\title{
Successful implementation of strategies to transform Emergency Department transfusion practice.
}

Matthew J Reed, Sarah-Louise Kelly, Hannah Beckwith, Catherine J Innes, Lynn Manson

\begin{abstract}
Blood component transfusion is an important and lifesaving Emergency Department (ED) procedure. It is not however risk-free and careful consideration of its clinical benefit for each individual patient is therefore essential. In 2008, we audited the patterns of blood component usage in 2007 within our ED. This work revealed that whilst 3209 units of blood component were ordered only $39.5 \%$ were transfused, and $9.5 \%$ were unaccounted for. This was the first and only published detailed look at ED blood transfusion practices. We had to address our poor traceability (i.e. unaccounted for units), our high blood usage, and our ordering of units which were then not transfused as this can lead to wastage. Firstly, better links between the ED and the Scottish National Blood Transfusion Service (SNBTS) were established. A set of improvement measures were then implemented including better ED medical and nursing staff education, monthly traceability reports sent to the ED clinical management teams, the introduction of an ED transfusion guideline, moving our blood fridge into the resuscitation room, having a named ED transfusion consultant and ED transfusion link nurse, ED consultant representation on the Hospital Transfusion Group and finally increasing awareness of ED emergency transfusion with a rotational thromboelastometry (ROTEM) research programme. In 2012, we reaudited our practice looking at our blood component usage in 2011 . There was a $64 \%$ reduction in blood component ordering (3209 vs. 1034 units), a $39 \%$ reduction in blood component transfusion (1131 vs. 687 units), a $68 \%$ increase in the proportion of ordered units that were transfused and a $96 \%$ reduction in unaccounted units (289 vs. 9 units) between 2007 and 2011. In attempting to cost the savings resulting from our changes we showed that SNBTS spent £306,437 less in 2011 compared to 2007 on handling and issuing ED transfusion requests. Our improvements are immediately generalizable across the UK and the potential savings to the NHS are enormous.
\end{abstract}

\section{Problem}

Blood component transfusion can be a vital and lifesaving intervention when given appropriately. However transfusion is not risk-free, and therefore careful consideration of its clinical benefit for each individual patient is essential. Supply of blood components is a finite resource, relying on continuous public donation to maintain adequate stocks, as components have a limited period of viability. Blood and its various components is therefore an extremely valuable commodity, requiring careful allocation to maximise clinical benefit and avoid wastage.

In 2008, we decided to look at our patterns of ED blood component use. This had not previously been studied in our ED, and more general information on the use of blood components and the characteristics of transfusion recipients was found to be limited [1-3]. A search of the literature using Medline revealed no information on the usage of blood components within UK EDs. We were also aware that in 2005, the 'Blood Safety and Quality' regulations were established stating that there must be full traceability of all aspects of the transfusion process from donor to recipient vein, maintained and available for 30 years [4]. Our performance in traceability had been poor over the previous couple of years however we had no information as to which components and which clinical conditions were involved. We also had no idea generally as to our pattern of blood component usage.

\section{Background}

The appropriate use of donor blood and its components, and effective alternatives is a much discussed public health issue, partly because of the increasing age of the population, the subsequent increase in demand for blood components and increasing costs of healthcare attributable to transfusion therapy [5-6]. In 2001, the National Blood Stocks Management project reported 2.2\% red cell concentrate (RCC) clinical wastage in $40 \mathrm{NHS}$ hospitals [7]. In the USA, Wallace et al reported that 1.8 million (13.5\%) of 13.3 million RCC units were wasted in 1994 (combined laboratory and clinical wastage) [8]. Reasons for this disparity may include differences in patterns of injury between the USA and UK or may reflect the improvements made in blood component utilisation over the intervening years. We were not able to find any information on other UK or international EDs' performance at tracing each blood component unit from donor to its final fate and therefore obviously no evidence for how to solve our potential poor traceability problem.

\section{Baseline measurement}

In September 2008, we performed a retrospective case note review to establish blood component usage and wastage within our ED in 2007 during which we had a total of 104,294 presentations. All adult patients ( $>12$ years of age) presenting to the ED for whom blood components were ordered were enrolled. Albumin orders were excluded as albumin is not ordered on a patient-specific basis. Routinely kept records of all blood components issued by the Royal Infirmary of Edinburgh (RIE) blood bank were obtained for the period 1 January 2007 to 31 December 2007. A data extraction 
form was designed and information regarding age, sex, type and amount of blood component, and indication for the transfusion was collected for all ED patients requiring blood components. Only patient-specific components were included in the study. If a patient had more than one reason for requiring a transfusion, all indications were documented. The outcome of the transfusion request was recorded as wasted (unit of blood component was not transfused to the patient for whom it was requested and was not returned to the blood bank in time for it to be recycled, and was wasted), recycled (unit of blood component was not transfused but was returned to the blood bank in adequate time to enable it to be recycled) or transfused (intended patient received that unit of requested blood component). Data were entered into a Microsoft Excel database (Microsoft Corporation, Redmond, Washington, USA) for statistical analysis. Results were analysed by individual episodes of transfusion. Median and interquartile range (IQR) were calculated for non-parametric data and mean and standard deviation for parametric data.

Between 1 January 2007 and 31 December 2007, 770 transfusion requests were identified, representing a total of 3209 units of blood components. After albumin was excluded, 758 case notes were searched for and 722 were successfully retrieved and reviewed (95.3\%). These 722 reviewed requests represented 550 patient episodes and 3048 requested units; some patients required more than one type of blood component and others presented frequently over the 1-year period. The median age of recipients was 65 (IQR 46-78) years and $56 \%$ of all transfusions were given to patients over 60 years old with an increase in transfusion rate across all age groups, continuing to 90 years and above. $57 \%$ of transfusion requests were for men [9].

Of the 3048 units requested, 97 were wasted (3.2\%) and 1458 $(47.8 \%)$ recycled; 1204 were transfused $(39.5 \%)$ leaving $289(9.5 \%)$ unaccounted for. The highest wastage rates (10\%) were seen with Fresh Frozen Plasma (FFP). Gastrointestinal bleeding was the most frequent indication for blood component usage with 5.2 units being requested per patient; a total of 1128 units of blood components. The highest mean number of units ordered per request was 6.0 units for abdominal aortic aneurysm (AAA). A total of 50 RCC units, 23 units of platelets and 24 units of FFP were wasted. Red Cell Concentrate (RCC) wastage was most frequently seen in patients presenting with alcoholic liver disease-associated bleeding.

See supplementary file: ds $1587 . j p g$

\section{Design}

After our initial review of ED blood component usage in 2007, we decided that our emergency transfusion performance was poor and we needed to implement improvement measures. We improved links between our ED and our Blood Transfusion Service (BTS) and then set out to implement a set of improvement measures. These measures included improved formal ED medical and nursing staff education with increased awareness of the mandatory Module 1 Safe Blood Transfusion which also highlights Medicines and Healthcare products Regulatory Agency (MHRA) traceability regulations where the importance of returning blood component tags is stressed, monthly traceability reports sent to the clinical management teams, the introduction of an ED departmental transfusion guideline (which includes guidance on which components to order for which conditions, how many and how quickly), refinements to the major transfusion protocol allowing faster access to emergency blood and having pre-thawed FFP available, moving our blood fridge into the resuscitation room, limiting the storage to emergency $O$ negative only (enabling easier access to the blood fridge meaning only one unit would be removed at a time during an emergency and making it easier for staff to record the removal of the unit on the satellite fridge register which completes the audit trail requirements), the introduction of a named transfusion consultant and transfusion link nurse in the ED (meaning staff know where to get advice if needed), ensuring ED consultant representation on the Hospital Transfusion Group, and finally increasing awareness of emergency transfusion by undertaking ED based research into Rotational Thromboelastometry (ROTEM) near-patient coagulation testing.

\section{Strategy}

Our main strategy revolved around improving links between key personnel in the ED and BTS. Key individuals in the ED of the RIE were identified at senior medical and nursing level. These individuals liaised with identified key individuals in the SNBTS of the RIE including a blood transfusion consultant and a transfusion practitioner. A named ED transfusion consultant and transfusion link nurse in the ED were also identified and ED consultant representation on the Hospital Transfusion Group was ensured. By identifying key links between both departments, it meant that a named individual in both departments was available for communication when any issues arose. This also allowed the ED medical and nursing teams to work effectively with each other and also with the SNBTS team. The identification of early goals of reducing blood component ordering and usage, and improving traceability meant also that both teams were able to work towards a shared target. It is apparent that this approach worked well and that the teamwork built up between both departments, and between the nursing and medical ED staff was a huge factor as the same improvements have not been apparent in any other departments in our hospital.

\section{Results}

In June 2012 we performed a second retrospective case note review looking at 2011 practice after our interventions. The hospital blood bank; RIE BTS (run by SNBTS) provided information on all blood components ordered and transfused by the ED in 2011, with corresponding patient hospital numbers. The Lothian Regional Ethics Committee deemed that the study was a service evaluation and therefore did not require formal ethical review. A data extraction form used previously for the baseline measurement was used to record information. One extraction form was used per patient episode where blood components were ordered. Individual Electronic Patient Records (EPRs) were accessed to obtain information on the ED admission history and indication for 
transfusion. Additional information including blood pressure and haemoglobin level at the time of the transfusion request was also recorded. The outcome of each transfusion request was recorded as transfused, recycled, discarded or unaccounted for. Data provided by the blood bank allowed transfused units to be recorded for specific patient episodes; however, discarded or unaccounted for blood component data was obtained from monthly figures, as this information was not available on an individual patient basis. Recycled units were those units returned to the blood bank with a secure 'cold chain' (within acceptable storage conditions while outwith blood bank control) and therefore able to be re-entered into stock for issue to another patient; discarded units were those units returned to blood bank whose cold chain was not secure and so could not be reissued. In cases where a unit of a blood component was issued by the blood bank and neither the unit nor the unitspecific identification tag was returned, this unit was recorded as unaccounted for.

Data was entered into a Microsoft Excel database (Microsoft Corporation, Redmond, Washington, USA) for statistical analysis. Results were analysed by individual episodes of transfusion. Median and IQR was calculated for non-parametric data and mean and SD for parametric data.

Between 1st January and 31st December 2011, blood components were requested for 255 patient episodes, totalling 1034 individual units. 687 units $(66.4 \%)$ of blood component were transfused, 248 components (24.0\%) were recycled, 90 components $(8.7 \%)$ were discarded and nine units $(0.9 \%)$ were unaccounted for.

There was a $64 \%$ reduction in blood component ordering (3209 vs 1034 units), a $39 \%$ reduction in blood component transfusion (1131 vs 687 units), a $68 \%$ increase in the proportion of ordered units that were transfused, and a $96 \%$ reduction in unaccounted units (214 vs 9 units) between 2007 and 2011 [10]. There was also interestingly, a rise in the median age of the patient for whom a transfusion request was made from 63.9 years in 2007 to 67.0 years in 2011 . Gastrointestinal (GI) bleeding was again the most common indication for transfusion with 116 requests (45.5\%).

See supplementary file: ds1590.jpg

\section{Lessons and limitations}

We have been able to demonstrate a $64 \%$ reduction in blood component ordering, a $39 \%$ reduction in component transfusion, a $68 \%$ increase in the proportion of ordered units that are transfused and a $96 \%$ reduction in unaccounted units, undoubtedly improving patient safety as a result of better emergency transfusion practice. These improvements are immediately generalizable across the UK. In addition we have attempted to cost the savings resulting from our changes. In Scotland, SNBTS, unlike NHSBT (National Health Service Blood and Transplant) in England and Wales, do not charge locally for blood components. We have therefore used charges to local private hospitals to estimate savings. In 2007, the total cost of 770 transfusion requests leading to the issue of 2879 units was estimated to be $£ 389,401$. $£ 183,920$ was the estimated unnecessary handling cost of units not transfused. In 2011 the total cost of 255 transfusion requests leading to the issue of 1034 units was estimated to be $£ 133,586$. $£ 43,001$ was the estimated unnecessary handling cost of units not transfused. Accounting for a $13 \%$ change in the value of the pound between 2007 and 2011, SNBTS spent $£ 306,437$ less in 2011 compared to 2007 on handling and issuing all transfusion requests, including $£ 159,238$ less on handling units not transfused. The fact that SNBTS are not able to use local financial incentives to drive down wastage and recycling, usually a very effective way of changing practice highlights just how good our strategies have been. The potential saving to the NHS is enormous.

This study does have some limitations. While we have observed a huge improvement in blood component ordering, usage and traceability since 2007 in our ED following implementation of simple strategies, we cannot prove that this implementation directly caused the improvement. The importance of $100 \%$ traceability on all blood components has been promoted greatly both locally and nationally by SNBTS and their many initiatives to improve staff education have most definitely contributed something to this improvement. The study was also retrospective in design; not all desired information was available for each patient episode and in some cases judgement had to be used when the indication for transfusion was unclear. However, data was available on the fate of every unit ordered by the ED during 2011 allowing our results to be a true representation of blood component usage in our ED.

We hope that with the identification of named ED transfusion link nurses and now excellent relationships between the ED and BTS, as well as a culture in our ED of blood transfusion audit and research, these improvements can be maintained and we can now focus on reducing wastage rates, which paradoxically, now we know what has happened to all of our units, have risen (previously these units were coded as unaccounted for). This will be tough, however since our review in June 2012 we have started to make inroads into this as well, using a similar ED teamwork approach to that demonstrated here.

\section{Conclusion}

Blood component ordering, usage and traceability within our ED have improved significantly since 2007 following implementation of simple strategies. We have been able to demonstrate a $64 \%$ reduction in blood component ordering, a $39 \%$ reduction in component transfusion, a $68 \%$ increase in the proportion of ordered units that are transfused and a $96 \%$ reduction in unaccounted units, undoubtedly improving patient safety and potentially saving money, as a result of better Emergency Transfusion practice. The age of ED transfusion recipients is increasing; this may have implications for future ED transfusion practice.

\section{References}

1. Vamvakas EC, Taswell HF. Epidemiology of blood transfusion. Transfusion 1994; 34: 464e70.

2. Chiavetta JA, Herst R, Freedman J, et al. A survey of red cell use in 45 hospitals in central Ontario, Canada. Transfusion 1996; 36: $699 \mathrm{e} 706$. 
3. Mathoulin-Pelissier S, Salmi LR, Verret C, et al. Blood transfusion in a random sample of hospitals in France. Transfusion 2000; 40: 1140e6.

4. The Blood Safety and Quality Regulations 2005.

http://www.opsi.gov.uk/si/si2005/20050050.htm

5. NHS Executive. Better blood transfusion: appropriate use of blood. London: Department of Health, 2002(Health Service Circular (HSC): 2002/009).

6. Wells AW, Mounter PJ, Chapman CE, et al. Where does blood go? Prospective observational study of red cell transfusion in north England. BMJ 2002;325:803e5.

7. Chapman J. The national blood stocks project. $\mathrm{Br} \mathrm{J}$ Haematology 2001;113 (Suppl 1):58.

8. Wallace EL, Churchill WH, Surgenor DM, et al. Collection and transfusion of blood and blood components in the United States. Transfusion 1998;38:625e36.

9. Beckwith H, Manson L, McFarlane C, Reed MJ. A review of blood product usage in a large Emergency Department over a oneyear period. Emerg Med J. 2010; 27: 439-442

10. Kelly S-L, Reed MJ, Innes CJ, Manson L. A review of blood component usage in a large UK emergency department after implementation of simple measures. Emerg Med J 2012; doi:10.1136/emermed-2012-201747

\section{Declaration of interests}

Nothing to declare

\section{Acknowledgements}

Thanks to Carol McFarlane for help with baseline measurement. Huge thanks are also required to Kate Mcllwraith and Rachel O'Brien, our ED transfusion link nurses and to the ED nursing and medical staff who have implemented these changes so effectively. 